\title{
Thioredoxin1 regulates conidia formation, hyphal growth, and trap formation in the nematode-trapping fungus Arthrobotrys oligospora
}

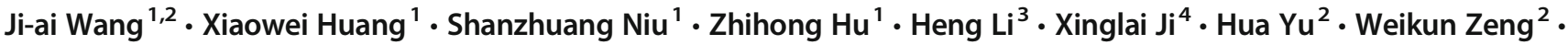 \\ Jian $\mathrm{Tao}^{2}$ - Weiwei Chen ${ }^{2}$. Jun $\mathrm{Li}^{2}$ • Juan $\mathrm{Li}^{1} \cdot$ Ke-Qin Zhang ${ }^{1}$
}

Received: 29 January 2019 / Accepted: 22 August 2019 / Published online: 29 August 2019

(C) Università degli studi di Milano 2019

\begin{abstract}
Purpose Arthrobotrys oligospora, a model nematophagous fungus that produces specific adhesive networks to capture nematodes, has been proposed as a potentially effective biological agent to control harmful plant-parasitic nematodes. Although thioredoxin has been characterized as playing important roles in many cellular processes in other species, its function in nematophagous fungi has not been studied. Here, the function of a thioredoxin homolog, Aotrx1, was investigated in A. oligospora.

Methods The encoding gene of Aotrx1 in the nematophagous fungus A. oligospora was knocked out by homologous recombination; strain growth was assessed.

Results The $\Delta$ Aotrxl strain of A. oligospora showed a significant decrease in growth rate on different media (PDA, CMY, and TG), a 70\% decrease of conidia production, and a lower germination rate compared with the wild type. The mutant strain was unable to form traps to capture nematodes and was more sensitive to SDS and $\mathrm{H}_{2} \mathrm{O}_{2}$.

Conclusion Thioredoxin is involved in conidia development, trap formation, normal mycelial growth, and resistance to environmental stresses in the nematode-trapping fungus A. oligospora.
\end{abstract}

Keywords Arthrobotrys oligospora $\cdot$ Thioredoxin1 $\cdot$ Conidiation $\cdot$ Nematicidal activity $\cdot$ Phenotypic characteristic

Ji-ai Wang and Xiaowei Huang contributed equally to this work.

Electronic supplementary material The online version of this article (https://doi.org/10.1007/s13213-019-01511-5) contains supplementary material, which is available to authorized users.

Juan Li

juanli@ynu.edu.cn

Ke-Qin Zhang

kqzhang@ynu.edu.cn

1 State Key Laboratory for Conservation and Utilization of Bio-Resources in Yunnan, Yunnan University, Kunming 650091, People's Republic of China

2 School of Medicine, Kunming University, Kunming 650214, People's Republic of China

3 Institute of Medical Biology, Chinese Academy of Medical Sciences \& Peking Union Medical College, Kunming 650118, China

4 Smart Health Big Data Analysis and Location Services Engineering Lab of Jiangsu Province, Nanjing University of Posts and Telecommunications, Nanjing 210023, China

\section{Introduction}

Thioredoxin (Trx) is a major multifunctional protein containing a conserved redox-active disulfide/dithiol sequence: Trp-Cys-Gly-Pro-Cys. This ubiquitous disulfide reductase serves as an electron donor for diverse enzymes (Arnér and Holmgren 2000). It can break disulfide bridges of target proteins and, rarely, promote the formation of disulfide bridges (Sahrawy et al. 1996). Consequently, its expression can directly cause the reduction of disulfide groups in proteins (Belozerskaia and Gessler 2007). Together with thioredoxin reductase and NADPH, Trxs form the "thioredoxin system" that can balance the levels of sulfhydryl groups and disulfides to maintain redox homeostasis (Arnér and Holmgren 2000). The thioredoxin system is highly conserved and is involved in a variety of important physiological processes throughout prokaryotic and eukaryotic cells (Thon et al. 2007). In 1964, Trx was first discovered in Escherichia coli, acting as an electron donor for ribonucleotide reductase (Laurent et al. 1964). In the pathogenic microorganism Cryptococcus neoformans, Trx 
was found to contribute to virulence (Missall and Lodge 2005). In mammalian cells, the lack of cytosolic Trx causes embryonic death. In addition, Trx is known to play important roles in defense against oxidative stress (Arnér and Holmgren 2000); participates in programmed cell death (Ravi et al. 2005), inflammatory responses (Nakamura et al. 2005), lifecycles of viruses and phages (Holmgren 1989), and breast cancer development (Bhatia et al. 2016); and acts as an Sdesulfhydrase (Collet and Messens 2010).

Previous studies of Trx in the model fungus Aspergillus nidulans demonstrated that deletion of the encoding gene tr $x A$ caused decreased vegetative growth, increased catalase activity, and failure to form reproductive structures such as conidiophores or cleistothecia (Thon et al. 2007). Interestingly, in Beauveria bassiana, the yield of conidia was found to decrease by $42 \%$ in a tr $x 2$ deletion strain but increase by $21 \%$ in a $\operatorname{tr} x 4$ deletion strain (Zhang et al. 2015).

In this study, we were interested in the potential roles of Trx in Arthrobotrys oligospora, a widely distributed nematodetrapping fungus which produces adhesive networks to capture nematodes. The gene encoding thioredoxin 1 (Aotrxl) was knocked out of $A$. oligospora by homologous recombination. The knockout strain showed significant changes in many morphological and physiological characteristics, such as conidia yield, hyphal growth, trap formation, and nematicidal activity, suggesting important roles of Trx 1 in A. oligospora.

\section{Materials and methods}

\section{Strain and growth conditions}

The wild-type (WT) strain of A. oligospora (ATCC24927) was purchased from the American Type Culture Collection and maintained on potato dextrose agar (PDA). This fungus was originally isolated from soil in Sweden and provided to ATCC by Nordbring-Hertz. The nematode Caenorhabditis elegans was maintained in oatmeal water agar medium.

\section{Gene knockout of Aotrx1 from A. oligospora}

The A. oligospora gene Aotrx-1 (GenBank XM_011125943.1) was knocked out by using a gene replacement method described previously (Colot et al. 2006). The hygromycin cassette $(h p h)$ was obtained from plasmid pSCN44 using primers hph-F and hph-R (Staben et al. 1989). Partial upstream and downstream Aotrxl sequences were amplified using primers Aotrx 1-5F/5R and Aotrx 1-3F/ $3 \mathrm{R}$ (Table S1). These three fragments were inserted into the yeast shuttle vector pRS426 (a gift from Prof. KA Borkovich, University of California) (Christianson et al. 1992). Then, the PCR fragment containing the gene replacement cassette was introduced into protoplasts of the WT A. oligospora strain and hygromycin-resistant transformants were selected (Margolin et al. 1997). PCR and Southern blotting were used to confirm the Aotrxl deletion.

\section{Comparison of growth rates between the WT and $\Delta$ Aotrx 1 strains}

The WT and $\Delta$ Aotrxl strains were incubated on PDA (200 g/ L potato, $20 \mathrm{~g} / \mathrm{L}$ dextrose, $20 \mathrm{~g} / \mathrm{L}$ agar), TYGA $(10 \mathrm{~g} / \mathrm{L}$ tryptone, $5 \mathrm{~g} / \mathrm{L}$ yeast extract, $10 \mathrm{~g} / \mathrm{L}$ glucose, $5 \mathrm{~g} / \mathrm{L}$ molasses, $20 \mathrm{~g} / \mathrm{L}$ agar), CMY $(20 \mathrm{~g} / \mathrm{L}$ maizena, $5 \mathrm{~g} / \mathrm{L}$ yeast extract, $20 \mathrm{~g} /$ $\mathrm{L}$ agar), and $\mathrm{TG}(10 \mathrm{~g} / \mathrm{L}$ tryptone, $10 \mathrm{~g} / \mathrm{L}$ glucose, $20 \mathrm{~g} / \mathrm{L}$ agar $)$ media, respectively. Colony morphology and growth rate were observed for 7 days.
A

a

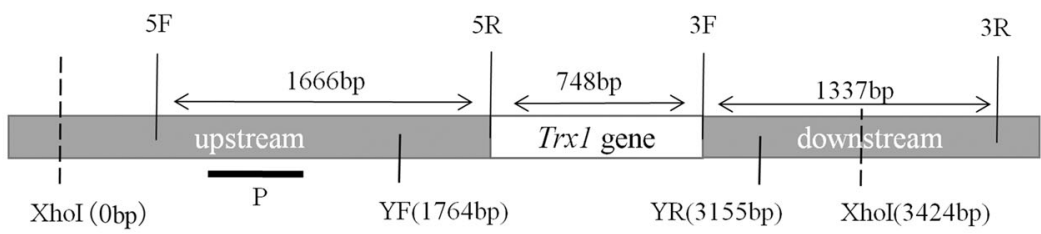

b

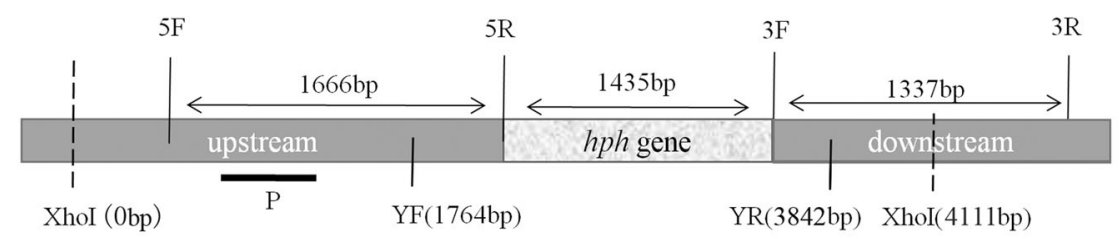

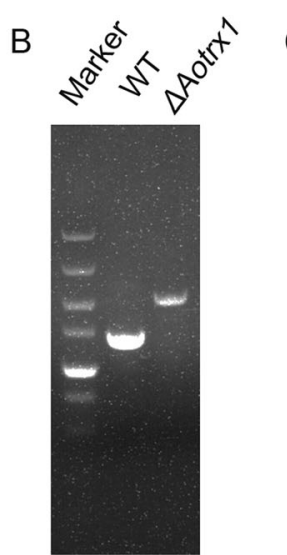

C
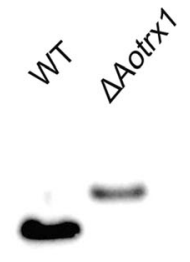

Fig. 1 Knockout and verification of Aotrxl deletion in Arthrobotrys oligospora. A Illustration of the method for replacement of Aotrxl. Primers $5 \mathrm{~F} / 5 \mathrm{R}$ and $3 \mathrm{~F} / 3 \mathrm{R}$ were used to amplify $5^{\prime}$ - and $3^{\prime}$ - flanking fragments of Aotrxl, and YF/YR were used for identification of mutants. $\mathrm{P}$ indicates the probe and $\mathrm{XhoI}$ was the endonuclease used for Southern blot analysis. a The wild type (WT); b the mutant. B Verification of Aotrxl deletion by PCR. The 2078-bp fragment proved the success of the Aotrxl knockout (WT 1391 bp). C Southern blot confirming the knockout in $\Delta$ Aotrx 1 

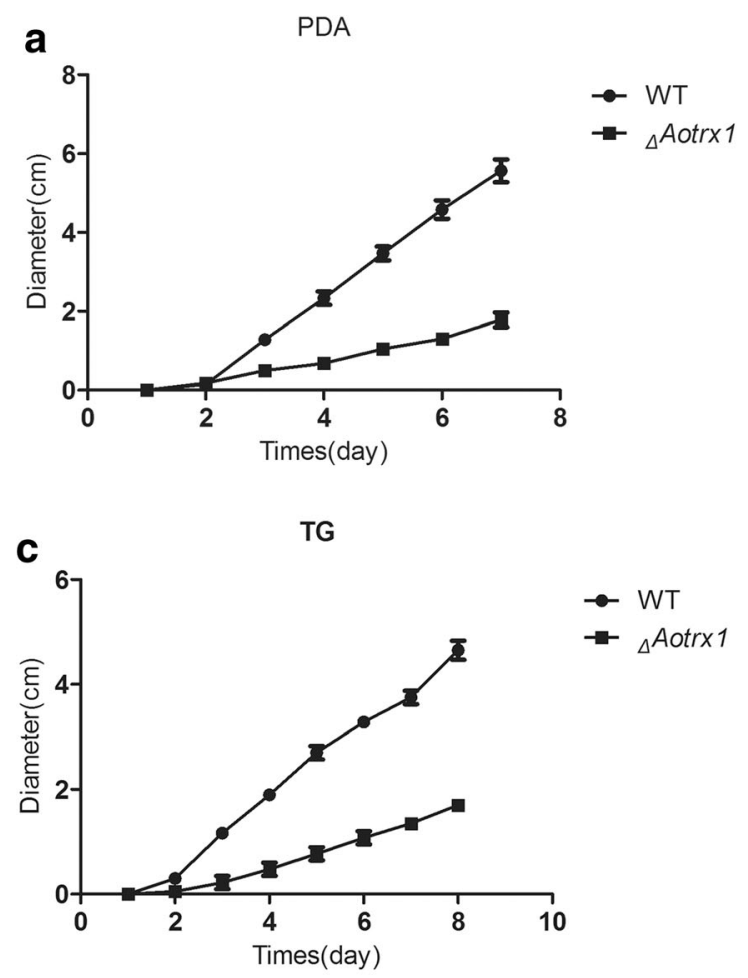

e

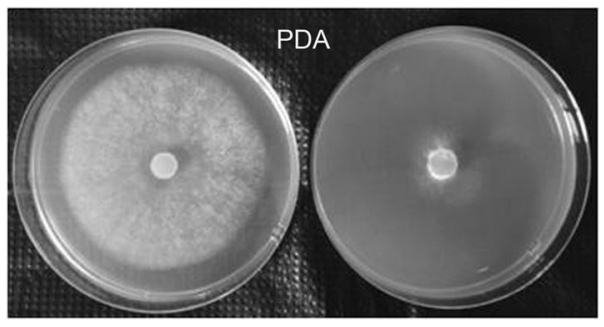

WT

$\Delta$ Aotrx1

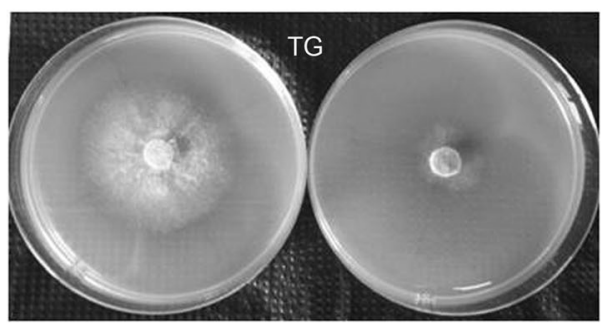

WT

$\Delta$ Aotrx1

Fig. 2 Decreased growth rate of strain $\triangle$ Aotrxl compared with WT. a-c $\triangle$ Aotrxl did not grow well on PDA, CMY, and TG media. $\mathbf{d}$ There was little impact on growth of the Aotrxl deletion strain on TYGA medium. e

\section{Comparison of conidia yield between the WT and $\Delta$ Aotrx 1 strains}

The WT and $\Delta$ Aotrxl strains were respectively incubated on $\mathrm{CMY}$ medium at $28^{\circ} \mathrm{C}$ for 15 days, and then, mycelia with the
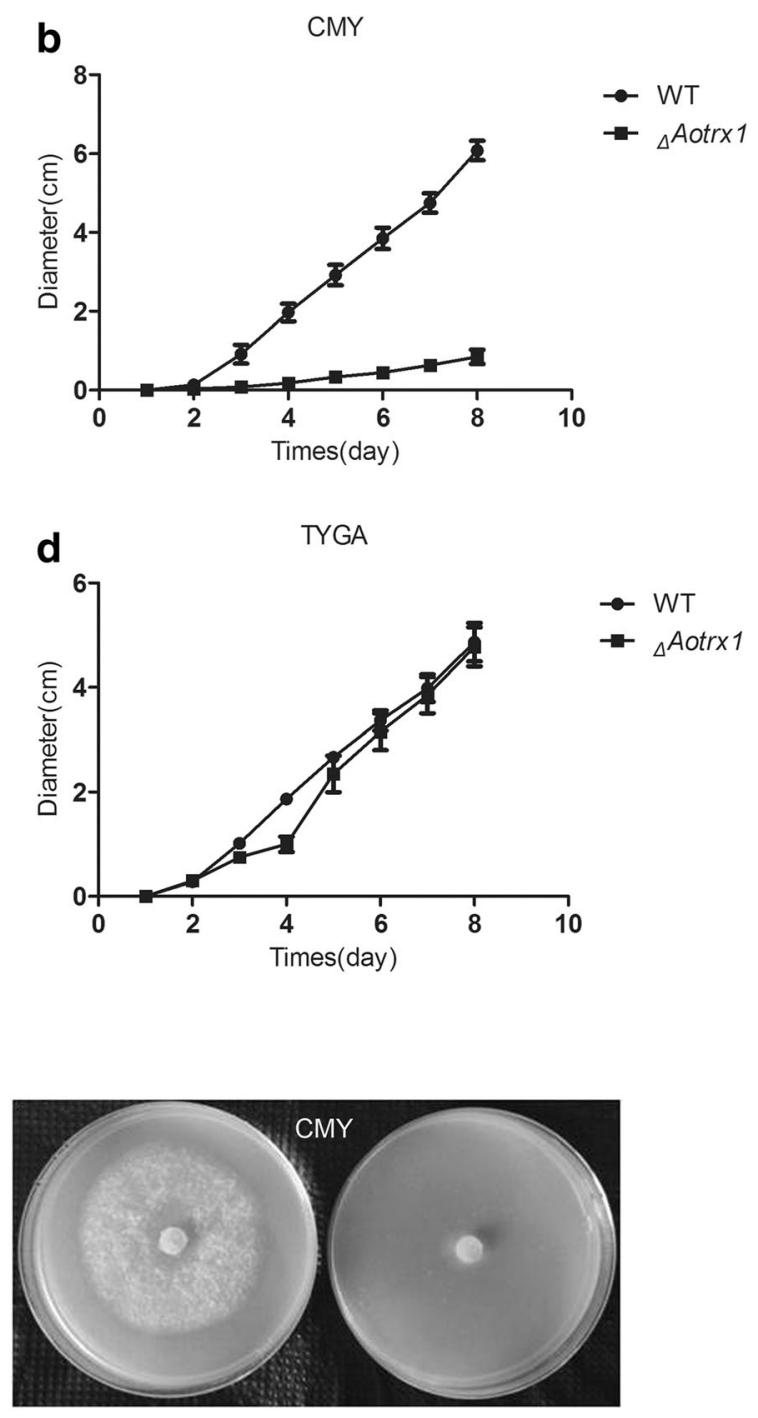

WT $\triangle$ Aotrx1

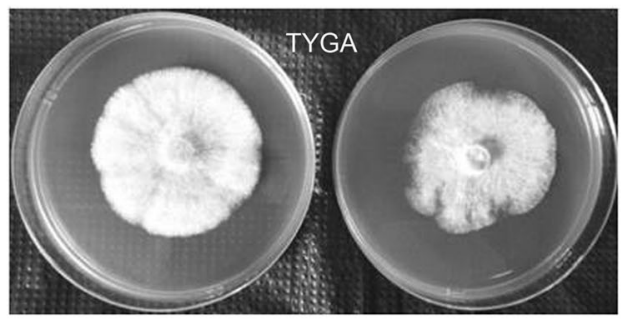

WT

$\triangle$ Aotrx1

Morphological features of aerial hyphae of $\Delta$ Aotrxl and WT strains on different growth media

same biomass were collected and filtered through six layers of lens tissue to collect the conidia. The conidia in suspension were counted using a hemocytometer (Xie et al. 2012). The germination rate of conidia and the mycelial morphology were characterized at 24 and $48 \mathrm{~h}$. 


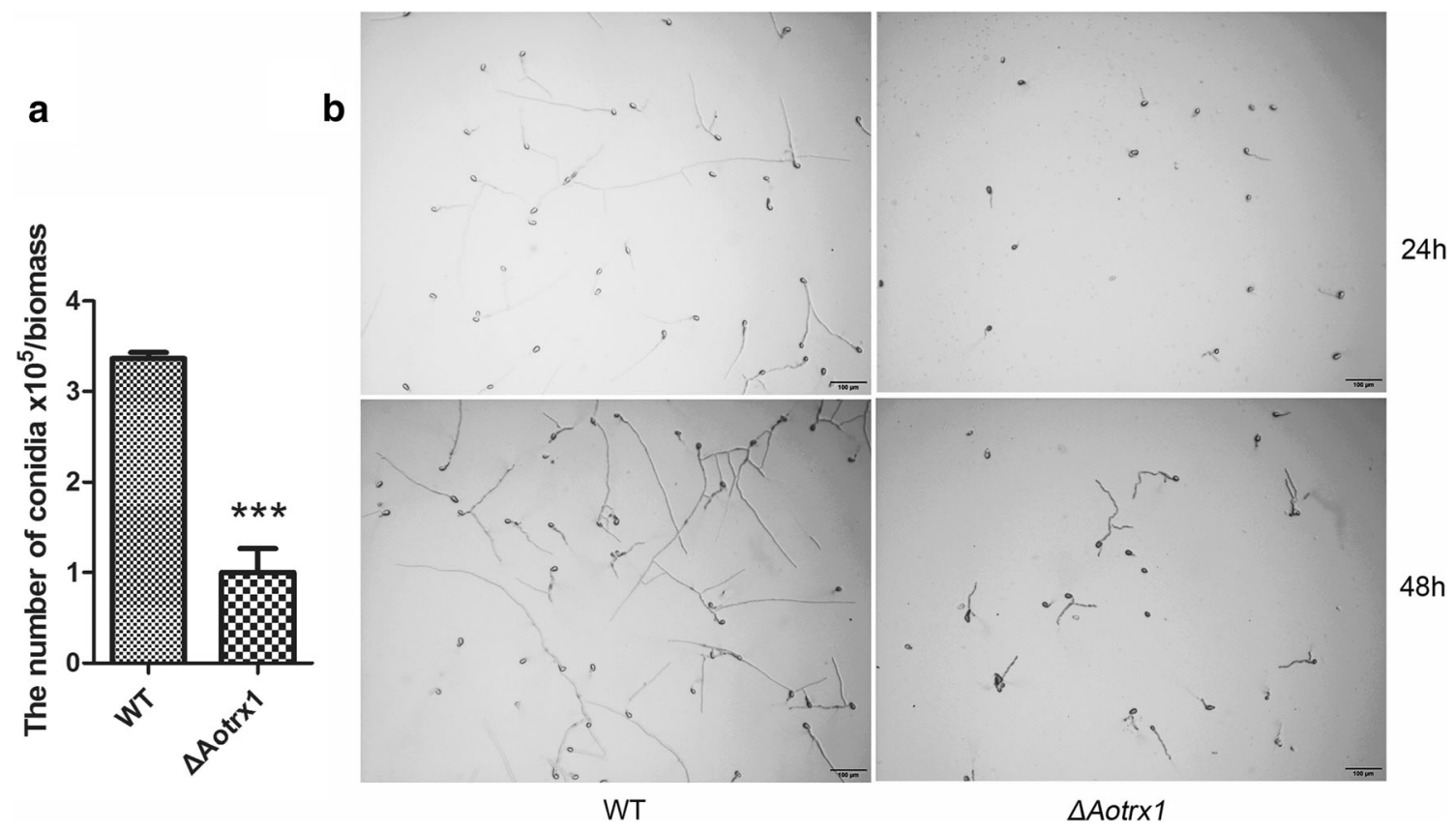

Fig. 3 Conidiation and morphological characteristics of spore germination. a Conidiation of the mutant showed an obvious decrease compared with the WT strain $(P<0.001)$. b Germination of spores at 24 and $48 \mathrm{~h}$, which showed the lower ability of strain $\Delta$ Aotrxl

\section{Induction of trap formation}

To analyze trap formation ability, both urea and nematodes were employed to induce traps. Firstly, conidial suspensions of the WT and $\Delta$ Aotrxl strains were spread over a water agar plate with urea $(500 \mathrm{mg} / \mathrm{L})$ and then induced at $28{ }^{\circ} \mathrm{C}$ for 16 days. In addition, approximately $10^{4}$ conidia of the WT and $\triangle$ Aotrxl strains were inoculated on CMA medium and incubated at $28^{\circ} \mathrm{C}$. After $4-5$ days of growth, about 200 adult nematodes were added to the $A$. oligospora mycelia. The traps were counted at specified time intervals using a light microscope (Olympus, Tokyo, Japan).

\section{Effect of different stress conditions on the growth of the WT and $\Delta$ Aotrx 1 strains}

To determine whether Trx plays a role in oxidative and detergent stress tolerance in A. oligospora, fresh mycelial plugs of WT and $\triangle$ Aotrx 1 were inoculated onto TYGA containing 5, 10 , and $15 \mathrm{mM} \mathrm{H}_{2} \mathrm{O}_{2}$, and $0.01,0.02$, and $0.03 \%$ SDS, respectively. The diameters of colonies were measured on the seventh day.

\section{Statistical analysis}

Three biological replicates were performed for each experiment, and all data were analyzed using GraphPad Prism version 5 (GraphPad Software, USA). $P$ values $<0.05$ were considered significant. Error bars indicate standard deviations (SD).

\section{Results}

\section{Knockout and verification of the deletion of the Aotrx 1 gene from A. oligospora}

pRS426 containing the $h p h$ gene as well as partial upstream and downstream sequences of Aotrxl was transformed into protoplasts of A. oligospora (Fig. 1a). After growing on selective medium, nine hygromycin-resistant clones were obtained (Fig. S1A). Compared with a 1391-bp fragment from the WT strain, a 2078-bp fragment was amplified from one transformant using primers YF/YR (Fig. $1 \mathrm{~b}$ and Table S1). Sequencing analysis of the PCR product confirmed that the Aotrxl genetic locus was successfully replaced by the hygromycin cassette (Fig. S1B). Southern blot analysis using probe $\mathrm{P}$ and restriction enzyme $\mathrm{XhoI}$ detected single bands in both the WT strain and the transformant, and the sizes were consistent with the predicted values (Fig. 1c).

\section{Effect of Aotrx1 mutation on hyphal growth}

To examine whether the disruption of Aotrxl influenced the growth of A. oligospora, we selected different media including PDA, CMY, TYGA, and TG to culture the WT and $\triangle$ Aotrxl strains. Compared with the WT strain, $\triangle$ Aotrxl showed a significant decrease in growth rate on PDA, CMY, and TG media (Fig. 2a-c), but a relatively small difference was observed on TYGA medium (Fig. 2d). After 7 days of incubation, the colony diameters of the WT strain were up to $6 \mathrm{~cm}$, while those of the $\Delta$ Aotrx 1 strain were $<2 \mathrm{~cm}$ on PDA 
Fig. 4 Trap formation and nematicidal activity. a Trap formation from spores of WT A. oligospora and the $\Delta$ Aotrx 1 mutant at $65 \mathrm{~h}$ and 16 days after urea induction. No traps were observed for the Aotrxl mutant strain, while the WT formed traps by $65 \mathrm{~h}$. b Spores of the $\Delta$ Aotrx 1 strain did not form traps when induced by C. elegans for $20 \mathrm{~h}$. Nematodes were still alive around $\triangle$ Aotrxl hyphae, while those around the WT strain of A. oligospora were killed and digested. The arrows indicate the three-dimensional traps a

WT

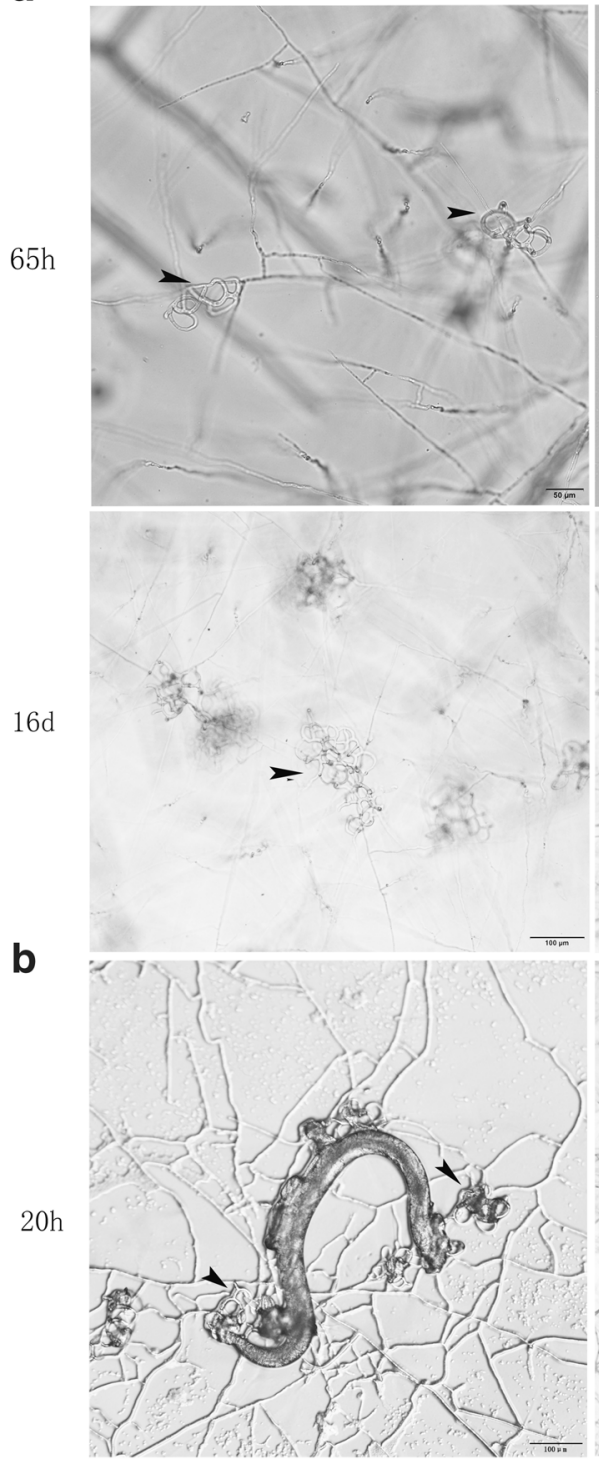

WT
$\Delta \operatorname{Aotrx} 1$

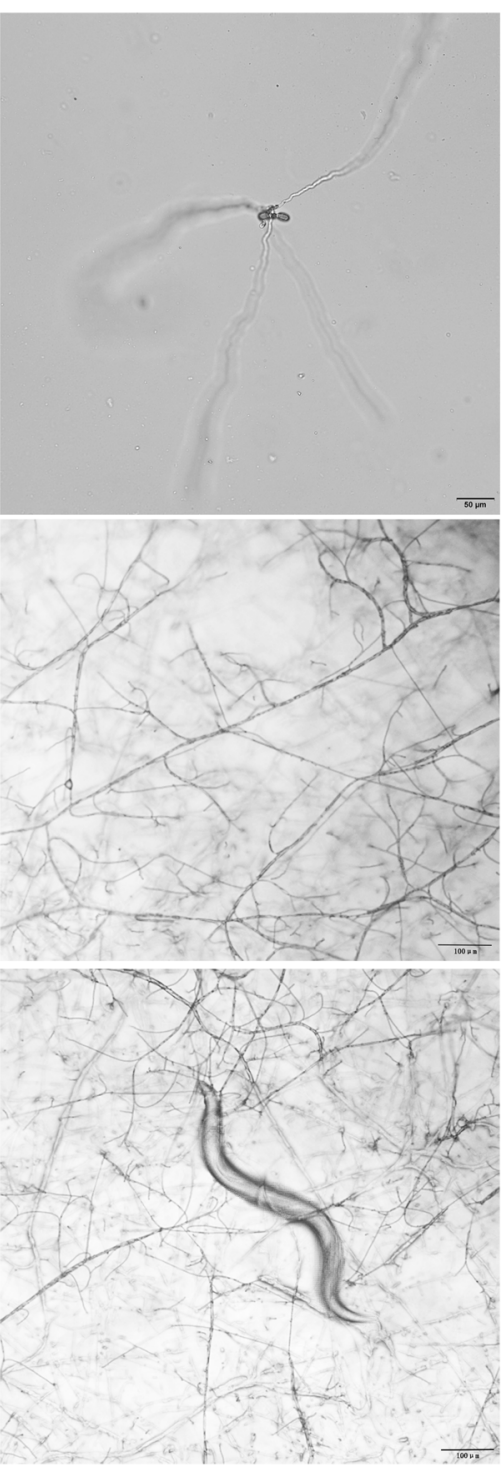

$\triangle \operatorname{Aotrx} 1$
(Fig. 2a), about $1 \mathrm{~cm}$ on CMY (Fig. 2b), and $2 \mathrm{~cm}$ on TG medium (Fig. 2c). Moreover, the aerial hyphae of $\Delta$ Aotrxl were sparser, and the colonies were less cottony and thicker than those of the WT strain (Fig. 2e).

\section{Effect of Aotrx 1 mutation on conidiation, trap formation, and nematicidal activity}

Compared with conidiation of the WT strain $\left(3.36 \times 10^{5} /\right.$ biomass), the production of conidia in A. oligospora was severely decreased by disruption of the Aotrxl gene $\left(1.01 \times 10^{5} /\right.$ biomass) (Fig. 3a). Moreover, the Aotrxl deletion strain also showed a lower germination rate than the WT strain (Fig. 3b).

To investigate the ability of Aotrx 1 to form nematode traps, both urea and the nematode $C$. elegans were used to induce three-dimensional adhesive networks in the WT and mutant strains with the same hyphal density. After induction with urea for $65 \mathrm{~h}$, the WT formed traps. However, no trap was observed in the $\Delta$ Aotrxl strain (Fig. 4a). Similarly, the WT strain formed many adhesive networks to capture nematodes within $20 \mathrm{~h}$, but the nematodes were still alive in the Aotrxl mutant test, which means that the nematicidal activity of strain $\Delta$ Aotrxl was also reduced (Fig. 4b).

\section{Effect of SDS and $\mathrm{H}_{2} \mathrm{O}_{2}$ stress on the growth of the WT and $\triangle$ Aotrx 1 mutant}

The growth rate and colony morphology of the WT and the $\Delta$ Aotrxl mutant were compared on TYGA medium with different stress factors (Fig. 5a and b). The mutant exhibited significantly slower growth rates than the WT strain on 
a CK

b

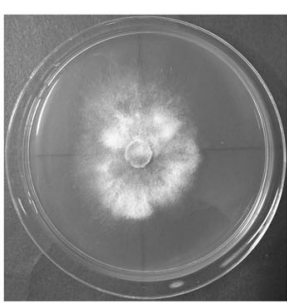

$\triangle$ Aotrx1
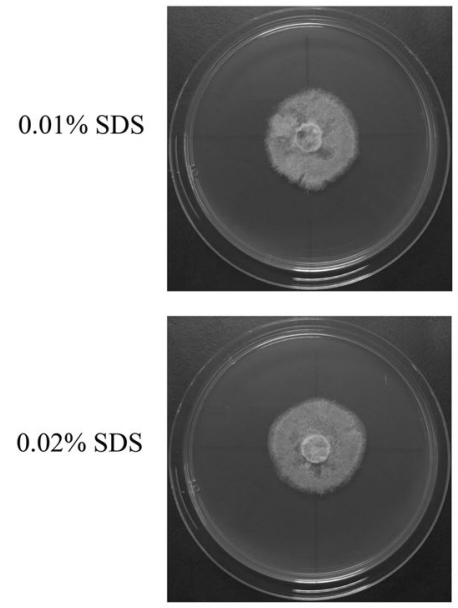

$0.02 \%$ SDS

$0.01 \%$ SDS

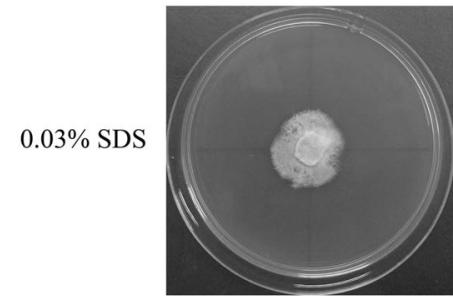

$\triangle$ Aotrx1

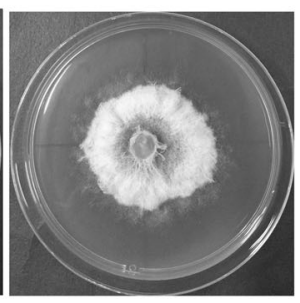

WT
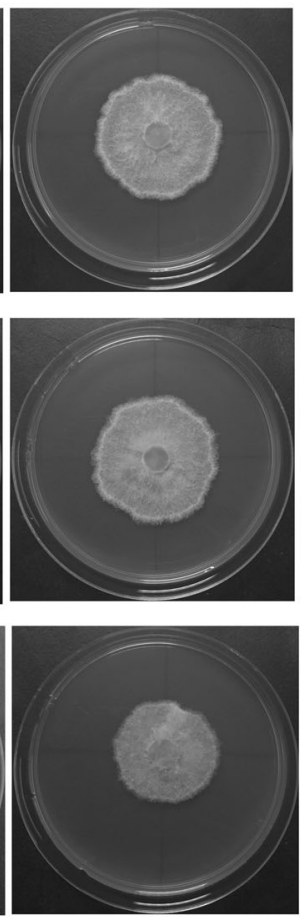

WT
Fig. 5 Stress tolerance. a Mycelial growth of WT and the $\Delta$ Aotrxl knockout strain showed no significant difference on TYGA medium. b Colony morphology of $\triangle$ Aotrxl and WT strains on TYGA medium

medium containing $0.01-0.03 \%$ SDS and could not grow on medium containing $5-15 \mathrm{mM} \mathrm{H}_{2} \mathrm{O}_{2}$ (Fig. $5 \mathrm{~b}$ and c).

\section{Discussion}

Trx is extensively distributed from archaea to human and is involved in multiple cellular roles (Arnér and Holmgren 2000). In this study, we characterized the function of a homo$\log$ of the Trx encoding gene, Aotrxl, in the nematophagous fungus $A$. oligospora. Compared with the WT strain, conidia production in the Aotrxl deletion strain decreased by $70 \%$, and the germination rate also decreased, suggesting that Aotrx 1 may be essential for the normal morphological development of conidia and mycelium. It has been reported that the trxA deletion strain of Aspergillus nidulans is unable to form
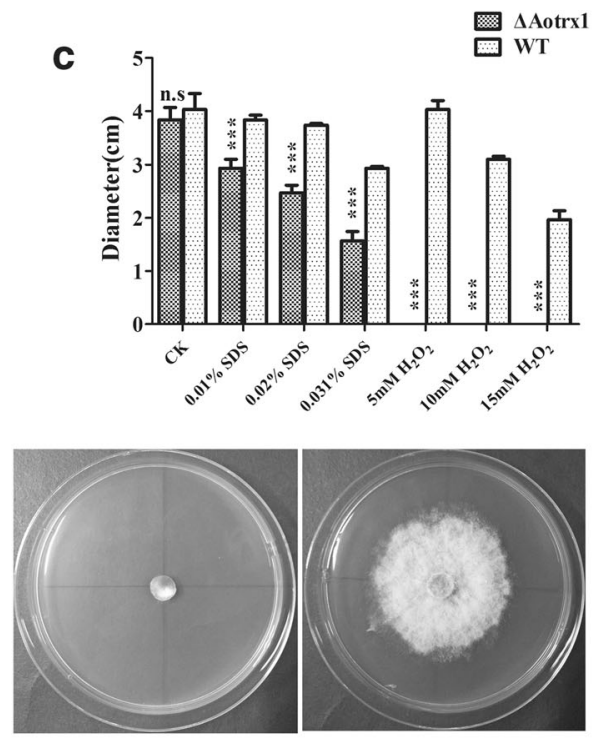

$5 \mathrm{mM} \mathrm{H}_{2} \mathrm{O}_{2}$
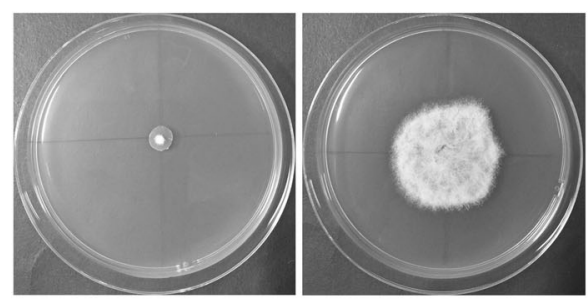

$10 \mathrm{mM} \mathrm{H}_{2} \mathrm{O}_{2}$

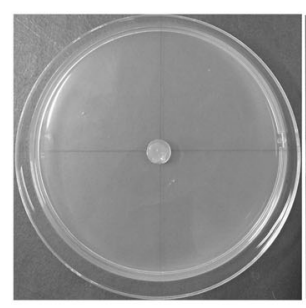

$\triangle$ Aotrx1

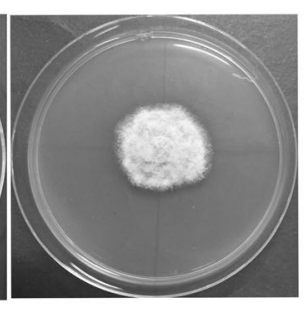

WT containing sodium dodecyl sulfate (SDS) or $\mathrm{H}_{2} \mathrm{O}_{2}$. c Statistics on colony diameter of $\triangle$ Aotrxl and WT strains on TYGA medium containing different concentrations of SDS or $\mathrm{H}_{2} \mathrm{O}_{2}$

conidiophores (Thon et al. 2007), while in B. bassiana, the yield of conidia decreased by $42 \%$ in a trx 2 deletion strain, but increased by $21 \%$ in a trx 4 deletion strain (Zhang et al. 2015). Our results and those of others suggest that Trx plays different roles in different fungal species.

Previous studies have shown that bacteria can release urea to trigger nematode-trapping fungi to form traps to capture nematodes. The deletion of genes related to urea transportation and metabolism abolished urea-inducible trap formation in A. oligospora (Wang et al. 2014). Surprisingly, the $\Delta$ Aotrxl mutant was unable to produce traps and kill nematodes in the presence of urea. This result suggests that Aotrx 1 may participate in some process(es) related to urea transport and metabolism. The fact that the $\Delta$ Aotrxl mutant is incapable of forming traps in the presence of $C$. elegans also suggests that Aotrxl is 
likely involved in cell wall biogenesis (Yang et al. 2011). Moreover, it showed inhibitory effects on the mycelial growth of the $\Delta$ Aotrxl strain in a cell wall-perturbing agent (SDS), suggesting that Aotrx 1 likely participates in cell wall synthesis by regulating the expression of other genes essential for nematicidal activity of A. oligospora. In addition, Trx has been reported to reduce $\mathrm{H}_{2} \mathrm{O}_{2}$ to $\mathrm{H}_{2} \mathrm{O}$ in many organisms (Arnér and Holmgren 2000). In our experiments, tolerance of the Aotrxl mutant to $\mathrm{H}_{2} \mathrm{O}_{2}$ was almost abolished compared with the WT implying the vital role of the thioredoxin system in the cellular response to oxidative stress.

Aside from the processes described above, our results are also consistent with Trx regulating other processes in A. oligospora, as found previously in other organisms. In the Aotrxl deletion strain, aerial hyphae were sparser, the mycelial growth rate decreased significantly, and the colonies were less cottony and thicker on PDA, CMY, and TG media, which is similar to the phenotype of the trx 1 mutant of C. neoformans (Missall and Lodge 2005) and the $\operatorname{tr} x A$ deletion strain of $A$. nidulans (Thon et al. 2007). However, our results were inconsistent with those in the model yeast Saccharomyces cerevisiae, which suggested that the thioredoxin system is not essential in normal growth conditions (Grant 2001). Here, a relatively small phenotypic effect of $\Delta$ Aotrxl was observed on TYGA, a nutrient-rich medium. Therefore, Trx may provide some important intermediates for normal fungal growth in the case of nutrient limitation.

Based on our experimental results, Trx is a multifunctional regulator of various physiological processes during asexual reproduction of A. oligospora. The regulated phenotypic traits include conidiation, mycelial morphology, spore germination, and trap formation. Trx participates in normal mycelial growth, resistance to environmental stresses, and nematicidal activity. At present, it is not known how Trx regulates these processes. In addition, the functions of other Trxs in this fungus are not clear. Regardless, our results clearly demonstrate the importance of Aotrxl in the nematodetrapping fungus $A$. oligospora.

Funding information This work is jointly funded by the National Natural Science Foundation of China (approved nos. 31,560,025, 31,460,024, $31,760,538,31,160,021,31,270,131$ and U1502262), the Department of Science and Technology of Yunnan Province (2017HB006), as well as the Yunnan Local Colleges Applied Basic Research Projects (2017FH001-113).

\section{Compliance with ethical standards}

Conflict of interest The authors declare that they have no conflict of interest.
Research involving human participants and/or animals N/A

Informed consent N/A

\section{References}

Arnér ES, Holmgren A (2000) Physiological functions of thioredoxin and thioredoxin reductase. FEBS J 267:6102-6109

Belozerskaia TA, Gessler NN (2007) Reactive oxygen species and the strategy of the antioxidant defense in fungi: a review. Prikl Biokhim Mikrobiol 43:565-575

Bhatia $\mathrm{M}$ et al (2016) The thioredoxin system in breast cancer cell invasion and migration. Redox Biol 8:68-78. https://doi.org/10.1016/j. redox.2015.12.004

Christianson TW, Sikorski RS, Dante M, Shero JH, Hieter P (1992) Multifunctional yeast high-copy-number shuttle vectors. Gene 110:119-122

Collet JF, Messens J (2010) Structure, function, and mechanism of thioredoxin proteins. Antioxid Redox Signal 13:1205-1216

Colot HV et al (2006) A high-throughput gene knockout procedure for Neurospora reveals functions for multiple transcription factors. Proc Natl Acad Sci U S A 103:10352-10357

Grant CM (2001) Role of the glutathione/glutaredoxin and thioredoxin systems in yeast growth and response to stress conditions. Mol Microbiol 39:533-541

Holmgren A (1989) Thioredoxin and glutaredoxin systems. J Biol Chem 264:13963-11396

Laurent TC, Moore EC, Reichard P (1964) Enzymatic synthesis of Deoxyribonucleotides. Iv. Isolation and characterization of thioredoxin, the hydrogen donor from Escherichia Coli B. J Biol Chem 239:3436-3444

Margolin B, Freitag M, Selker E (1997) Improved plasmids for gene targeting at the his-3 locus of Neurospora crassa by electroporation. Fungal Genet Rep 44:34-36

Missall TA, Lodge JK (2005) Function of the thioredoxin proteins in Cryptococcus neoformans during stress or virulence and regulation by putative transcriptional modulators. Mol Microbiol 57:847-858. https://doi.org/10.1111/j.1365-2958.2005.04735.x

Nakamura T, Nakamura H, Hoshino T, Ueda S, Wada H, Yodoi J (2005) Redox regulation of lung inflammation by thioredoxin. Antioxid Redox Signal 7:60-71

Ravi D, Muniyappa H, Das KC (2005) Endogenous thioredoxin is required for redox cycling of anthracyclines and p53-dependent apoptosis in cancer cells. J Biol Chem 280:40084-40096. https://doi. org/10.1074/jbc.M507192200

Sahrawy M, Hecht V, Lopez-Jaramillo J, Chueca A, Chartier Y, Meyer Y (1996) Intron position as an evolutionary marker of thioredoxins and thioredoxin domains. J Mol Evol 42:422-431

Staben C, Jensen B, Singer M, Pollock J, Schechtman M, Kinsey J, Selker E (1989) Use of a bacterial hygromycin B resistance gene as a dominant selectable marker in Neurospora crassa transformation. Fungal Genet Newsl 36:79-81

Thon M, Al-Abdallah Q, Hortschansky P, Brakhage AA (2007) The thioredoxin system of the filamentous fungus Aspergillus nidulans: impact on development and oxidative stress response. J Biol Chem 282:27259-27269. https://doi.org/10.1074/jbc.M704298200

Wang X et al (2014) Bacteria can mobilize nematode-trapping fungi to kill nematodes. Nat Commun 5:5776

Xie XQ, Li F, Ying SH, Feng MG (2012) Additive contributions of two manganese-cored superoxide dismutases (MnSODs) to antioxidation, UV tolerance and virulence of Beauveria bassiana. PLoS One 7:e30298 
Yang JK et al (2011) Genomic and proteomic analyses of the fungus Arthrobotrys oligospora provide insights into nematode-trap formation. PLoS Pathog 7:e1002179. https://doi.org/10.1371/journal. ppat. 1002179

Zhang LB, Li T, Ying SH, Feng MG (2015) Subcellular localization of six thioredoxins and their antioxidant activity and contributions to biological control potential in Beauveria bassiana. Fungal Genet Biol 76:1-9

Publisher's note Springer Nature remains neutral with regard to jurisdictional claims in published maps and institutional affiliations. 